\title{
AMBIENTALIZACIÓN PEDAGÓGICA DEL CURRÍCULO ACADÉMICO
}

\author{
Róger Martínez Castillo \\ Profesor Catedrático de la Universidad de Costa Rica (UCR) \\ y Universidad Nacional (UNA). \\ San José, Costa Rica
}

Recibido 21-II-2007 • Aceptado 12-XII-2006

"Excelente maestro es aquel que, enseñando poco,
hace nacer en el alumno el gran deseo de aprender"

Arturo Graf.

Resumen: El sistema de desarrollo socio-económico dominante, con sus políticas neoliberales y enfoques mercado-céntricos contribuyen a agravar los problemas socio-ambientales. No hay conciencia política de que el propio modelo de desarrollo vigente es el que genera la degradación socio-ambiental. Este tipo de temas no ha sido fácilmente comprendido o aceptado en el ámbito educativo formal, debido a la resistencia de la educación oficial para integrarlos en sus marcos académicos.

Ante la crisis socio-económica y ambiental que vive nuestra sociedad, la educación ambiental representa una opción viable a la problemática general, para promover cambios sociales, nuevos conocimientos, permitir la participación ciudadana y un desarrollo sustentable, que estimulen una sociedad más justa, democrática y solidaria.

Palabras clave: Educación, sociedad, ambiente y pedagogía.

\section{Introducción}

En el transcurso de la historia, el ser humano ha desarrollado una capacidad de alterar el medio que le rodea, de forma premeditada o inconsciente. Los cambios y alteraciones que lentamente se producen en el medio natural, dependen de la forma en que la sociedad percibe su entorno, en los objetivos y aspiraciones que tiene en relación con el uso, modificación y adaptación del medio natural a sus propias necesidades e intereses.

Para comprender las interrelaciones (necesidades e intereses) que existen entre el ser humano y su medio natural, es necesario profundizar en la educación, que la sociedad mantiene con respecto al entorno donde realiza sus actividades vitales. El desarrollo socio-económico juega un rol importante en este proceso y la educación, como factor socio-cultural es el vehículo de transmisión de los conocimientos. En un mundo donde existen grupos culturales distintos y cada uno de ellos tiene su forma 


\begin{abstract}
The dominant socio-economical development system, with its neo-liberal policies and market centered approaches, contributes to aggravate the socio-environmental problems. There is no political awareness that the current development model itself is the one that generates the socio-environmental degradation. This type of subjects has not been easily understood or accepted in the formal educational field, due to the resistance of the official education to integrate them in their academic frameworks.

Regarding the socio-economical and environmental crisis that our society faces, environmental education represents a viable option towards the general problem by promoting social changes, producing new knowledge, allowing for social participation, and encouraging sustainable development actions, that stimulate a more democratic, fair, and supportive society.
\end{abstract}

Key words: Education, society, environment, and pedagogy. (diferente) de ver e interpretar el entorno que le rodea.

La cultura (y sus mecanismos de adaptación cultural), como forma de adaptación es la que destaca al homo sapiens de otros animales, entendida en sentido antropológico, como el conjunto de información y artefactos que se transmite de una población a otra y de una generación a otra, por vía no genética. De ahí, que nos resulta compleja esa sopa cultural de la humanidad. En ella, la educación es uno de los instrumentos más importantes de adaptación cultural. La paradoja es que esa misma capacidad humana para la cultura que nos ha puesto en un disparatero planetario, debe proporcionar salidas a la crisis mediante cambios culturales en los patrones de relación entre humanos y la biosfera. Por eso se invoca a la educación como el mecanismo de esperanza de cambio social por excelencia.

Los problemas ambientales actuales son un tema importante de preocupación de organismos internacionales, instituciones nacionales y locales. Temas como el crecimiento global de la población mundial y su relación con los agroecosistemas, la deforestación, la lluvia ácida, la capa de ozono, son objeto de preocupación de la humanidad; que cada vez más generalizada, considera que puede poner en peligro la continuidad de la especie humana sobre la tierra. Sin embargo, no se toma conciencia de que es el propio modelo (estilo) de desarrollo dominante, el marco en el que se generan los desequilibrios socio-ambientales. La dinámica del sistema socio-económico vigente, sus políticas desarrollistas y el enfoque mercado-céntricos agravan estos mismos problemas. El análisis de estos aspectos en el ámbito educativo no ha sido fácil, debido a la tradicional resistencia de la educación oficial, por considerar perspectivas difíciles de integrar en los marcos académicos y por el hecho de que la educación ambiental haya surgido fuera de su contexto. 


\section{Crisis global de desarrollo}

La crisis global procede de las relaciones entre nuestra especie y la biosfera a través de la creación de ecosistemas humanizados. Los ecosistemas humanizados son el resultado de un proceso histórico de evolución en el que las condiciones ambientales, los patrones de comportamiento humano y los rasgos e instituciones socio-culturales interaccionan de forma compleja. Con manifestaciones ambientales y sociales muy graves que van a empeorar en el futuro, a medida que nos acercamos a los limites de soporte de la tierra. Somos el cáncer planetario. Lo que está en peligro no es el planeta, sino el colapso de la biosfera o ecosistemas humanizados y nuestro propio futuro como especie. La aparición y evolución de ecosistemas humanizados en la historia terrestre esta afectada por tres grandes acontecimientos catastróficos:

- $\quad$ aparece la vida en la tierra,

- $\quad$ surge la atmósfera oxidante, subproducto del metabolismo fotosintético, catastrófico al introducir el elemento tóxico para los organismos; y

- explosión de la transmisión cultural humana y su transformación del mundo.

Ha habido varias razones o aspectos, por los cuales nos hemos metido como especie en la crisis ambiental, entre ellas:

- el papel de los humanos en la naturaleza, ha sido y es muy destructiva;

- la idea aristotélica de lo humano como centro de todo, separado de lo natural;

- el concepto antropocéntrico en la relación especie humana-naturaleza;

- estudio sistemático de las relaciones especie humana-biosfera; y

- la nueva relación de los humanos con la biosfera, que a partir de la revolución industrial, generó tremendos cambios en la acción y cosmovisión de la cultura occidental, estimulando impactos negativos sobre el ambiente natural.

El planteamiento de un nuevo análisis de la crisis, el de la historia ecológica de la humanidad o biohistoria, para comprender el origen de la crisis global, la situación actual y los mecanismos de adaptación cultural, pueden ser relevantes para el futuro. La especie humana se incluye en la trama de la vida y la pone en peligro actualmente.

Sobresalen tres rasgos ecológicos de nuestra especie:

- capacidad de intervención sobre el ambiente, propiciada por la evolución cultural: población, uso de energía y materias primas, impacto sobre la biosfera.

- unidad de adaptación no es la población; sino la humanidad entera. El establecimiento de un ecosistema mundial a partir de la interdependencia creciente: ecológica y económica en todo el mundo, manifiesta el carácter global de la biosfera humanizada.

- desarrollo tecnológico y social ha abierto una brecha entre dos grandes sectores de la humanidad: el mundo desarrollado (rico) y el mundo en desarrollo (pobre), a través de relaciones asimétricas, intercambios desiguales económico y tecnológicos, donde el desequilibrio socio-económico repercute sobre la biosfera humanizada y se reflejan en el ambiente como muy desestabilizadores.

Entre los rasgos de la crisis ambiental, sobresale el deterioro de los sistemas claves, soportes de la vida como la atmósfera, el mar, la tierra fértil. La posibilidad de destruir la vida (humana) en el planeta, es enorme nuestra ignorancia sobre la biodiversidad y la riqueza de formas de vida, que 
está siendo degradada minuto a minuto, a través de la destrucción de sus hábitats de vida. Se está atacando el núcleo de la biosfera, su memoria genética: la biodiversidad es paradójicamente el mayor capital para el desarrollo sustentable. Lo más significativo y peligroso por su carácter global es el cambio climático, a través del efecto de invernadero y destrucción de la capa de ozono. Se dá el aumento de dióxido de carbono, relacionado con el incremento de la temperatura, con efectos catastróficos para la salud humana: cáncer de la piel, cataratas, afecciones oculares, etc.

El impacto sobre la biosfera es cada vez más grave, abriéndose un futuro incierto ante la humanidad. Es la diferencia entre la conciencia de una crisis local, que se puede solucionar si se dejan de producir impactos locales y la crisis global en la que se esta tocando las entrañas de la biosfera. Otra característica es el carácter de crecimiento explosivo que tienen las relaciones con el medio: la población humana, el consumo de materias primas, la energía, que amenaza el sistema limitado del planeta. Refleja lo insustentable y acelerado de la crisis ambiental. En el mismo medio que se ha creado nos encontramos inadaptados. Se necesita que la sociedad se ocupe de la biosfera, a través de un proceso educativo, para poder entender la crisis ambiental y eliminarla.

Pero, la crisis no solo es ambiental, es social y económica, ello debido a que el desarrollo en los países pobres no está ocurriendo, sino que el abismo de la pobreza se está agigantando. Se intensifican los sistemas de explotación de los bienes del planeta, con graves consecuencias ambientales; pues, estos países tienen que usar sus ecosistemas frágiles y valiosos. Participando así, en la destrucción ambiental, la corrupción de los gobiernos, las compañías transnacionales, la ignorancia y pobreza de la población, el injusto comercio mundial, para mantener un estilo de vida confortable y derrochador en los países ricos. El problema de estilo de vida o desarrollo no está en estudio como problema entre los países ricos, ni en los congresos internacionales; siendo uno de los obstáculos más importantes para un desarrollo sustentable efectivo. La vida de altos estándares de unas minorías de personas de países ricos, en unas cuantas generaciones de la humanidad, se está llevando a cabo con el subsidio de las privaciones de la gran mayoría de la población mundial.

\section{Educación y sociedad}

La transición se caracteriza por la INFORMACIÓN, donde pasan los medios de comunicación, la enseñanza, industria del ocio, política, administración pública, actividades financieras, moda, diseño, innovaciones tecnológicas, etc; que parecen incontrolables e ingobernables.

La educación es un proceso de transmisión de conocimientos y actitudes, sirve como un instrumento básico para transmitir los rasgos fundamentales de la cultura, las técnicas vitales para la sociedad y un conjunto de actitudes, normas, valores, etc. La educación mercantil refleja intereses de grupos dominantes de la estructura prevaleciente de la sociedad. Debemos comprender cuál es el sentido y el papel, en este contexto histórico, de educación ambiental. La educación como proceso tiene un importante lugar en la transmisión del pasado y la consecución del futuro. El primer requisito para la solución de un problema es el reconocimiento de que el problema existe; o sea, la toma de conciencia de ello (Boyden, 1987).

El estudio de la historia humana en la educación (pública o privada) enfatiza su atención a las guerras, los cambios en la ciencia y tecnología, el crecimiento y crisis socio-económicas, culturales y las relaciones de poder político-administrativas; pero, no hay una historia ecológica universal que estudie el agotamiento, explotación, deterioro y crisis de los ecosistemas y su enfoque socio-histórico, mediante la relación entrópica, holística, sistémica, que esté 
ligada al tipo de desarrollo social, económico, político, cultural y ambiental.

Aquí se plantea la interacción e importancia de la educación ambiental y el aspecto del medio ambiente en un contexto de educación, relación que debe ser explícita académicamente para aplicar la sostenibilidad socio-natural como un proceso de concienzación educativa y cultural.

La educación formal ha perdido importancia relativa con el desarrollo exponencial de los medios de comunicación. Ejemplo, las inversiones anuales en publicidad (estatal y privada) superan al presupuesto de Educación Pública. Las modas profesionales, el auge de una educación mercantilizada es un serio obstáculo para la sustentabilidad.

La urgencia de los cambios necesarios en la enloquecida carrera mercantil que deteriora la vida del planeta, no tiene parangón en los esfuerzos a la transformación. nativa?

Llegará a tiempo la educación alter-

Entre los mecanismos, formas o experiencias que parecen más útiles en educación ambiental están, el comprender la necesidad de cambio y adquirir motivación, son requisitos necesarios para actuar y encontrar un consenso local, nacional, entre todos los sectores. Por sí misma la comprensión de la realidad no implica dar los pasos para transformarla. La transición para crear un mundo con una sociedad más en equilibrio con la biosfera humanizada, depende de nosotros, de comprender nuestro futuro común, basada en el cambio de las estructuras de relaciones y de actitudes y valores.

Sobresalen los proyectos o actividades interdisciplinarias y transversales dirigidas a las comunidades, centros educativos, al rescate de cuencas hidrográficas, de la biosfera y los recursos naturales; ello implica la aplicación de la educación ambiental: cambios de valores, conductas, comportamientos, estilos de vida. Se deben estimular los conocimientos básicos en todo tipo de educación (formal, informal) de manera gradual, en un proceso de concienzación, mediante técnicas audiovisuales aplicadas, trabajo con grupos organizados, diversos sectores laborales y sociales: etnias, mujeres, etc.

Entre los agentes de cambio que van a ser más importantes en la transformación a un mundo sustentable, están: la educación, medios de comunicación, ONGs, empresas, cambios tecnológicos, sociales y políticos. Todos y cada uno es importante, dado que la realidad es interdependiente y está entrelazado, todos los agentes son necesarios; quizás algunos jueguen un papel mas activo que otro:

- Educación: está en primera línea de batalla, ya que debe crear conciencia del problema ambiental, encontrar soluciones, estimular nuevos valores, comportamientos y actitudes.

- Medios de comunicación: no deben manipular la información, ni servir a intereses privados, deben de enseñar no-formalmente y sistemático, lo importante del orden natural y social.

- ONGs: deben dejar de ser centros de enriquecimiento de un grupo cerrado y buscar cambios no sólo culturales (mentales, valores), sino y políticos (cambio de formas y sistemas de vivir), creando espacios de participación y solución de los problemas ambientales.

- $\quad$ Empresas: deben tomar en cuenta el medio natural, al reciclar y reutilizar agroecosistemas. La economía debe estar regulada y aplicarse con conciencia, valorizando el elemento natural y social, no como mercancía; sino como su importancia para la vida misma.

- $\quad$ Cambios tecnológicos: buscar nuevas técnicas y herramientas de producción más aptas ambientalmente, sin perder el desarrollo necesario; evitando alterar el entorno natural.

- $\quad$ Cambios políticos: la actual forma de vivir (producir y consumir), refleja 
que el estilo y modelo es insostenible. Hacer cambios socio-culturales sustentables. Las políticos deben ser integradas, tomando en cuenta intereses socio-ambientales.

- Cambios sociales: la sociedad debe ser más justa y equitativa, se deben eliminar las injusticias sociales, económicas y ambientales (pobreza $\mathrm{y}$ riqueza extrema).

Pese a la escasa integración oficial de la educación ambiental al currículo, muchos educadores ponen en práctica diversidad de proyectos y acciones concretas relacionadas, tanto en aulas como en comunidades. Estas iniciativas se relacionan con procesos de innovación que se producen en el mundo educativo y que pretenden romper con los rasgos convencionales y arcaicos de un modelo escolar inadecuado para cumplir sus funciones en una sociedad moderna.

Estas experiencias manifiestan grandes dificultades para incorporar lo ambiental al currículo. Los diseños curriculares intentan incorporar la dimensión ambiental, manejando el concepto de transversalidad, como mecanismo de inclusión de contenidos ambientales en la organización de carácter más vertical de conocimientos de las áreas académicas. No es fácil la integración de ambos contenidos, se corre el riesgo de que hacer un añadido de aspectos ambientales a conocimientos convencionales de un área; o que el enfoque transversal pueda convertirse en un cajón de sastre, donde se mete aquello que no cabe en otros espacios mas académicos. De ahí, el interés de conseguir una verdadera integración de la educación ambiental en el currículo.

Con el proceso de educación ambiental, la sociedad genera conocimientos y experiencias alternativas, para interiorizarlos y traducirlos en nuevos comportamientos, valores y actitudes, que lo conduzcan a una mejor interacción social, económica y política con su ambiente y sociedad. La educación ambiental plantea un nuevo desarrollo socio-económico alternativo, un cambio estructural, una revolución, donde lo ambiental es eje de la autogestión local, desconcentración, participación activa, descentralización, democratización y transformación de las relaciones de poder y la economía de mercado sea regulado, donde el entorno predominante nacional e internacional y la economía de mercado tome en cuenta lo natural y social.

Las primeras iniciativas en la educación para afrontar los problemas ambientales surgen en otros ámbitos sociales, como organismos de la ONU, a través de foros, conferencias, seminarios y congresos internacionales, como: Estocolmo-1972, Tbilisi-77, París-82, Moscú-87; donde se establecen una serie de recomendaciones en relación con la educación ambiental. La UNESCO fue quien popularizó la educación ambiental, a niveles gubernamentales, de científicos y decisores.

La Conferencia de las Naciones Unidas sobre Medio Ambiente y Desarrollo celebrado en Río de Janeiro, Brasil, en 1992, profundizó el concepto de desarrollo sostenible, desencadenando un importante proceso mundial, para que los gobiernos y la sociedad se planteara más directamente su implicación en los aspectos ambientales. Aquí, el problema ambiental dejó de ser un tema elite, específico de un país o región y tomó un matiz mundial, popular.

En Río se suscribieron varios convenios y se emitieron declaraciones de acción global a nivel internacional: la AGENDA XXI; donde se propone una reorientación conceptual y práctica de la educación hacia el desarrollo sostenible.

Río-92 demostró, que es imposible abordar una acción reequilibradora internacional con los actuales mecanismos dominantes de toma de decisión y forma de vivir; por eso, es necesario una transición de cambios sociales, políticos, económicos y ambientales.

\section{Currículo y ambiente natural}

Sin embargo, la educación ambiental no se ha incluido ni aplicado como 
materia transversal en los nuevos diseños curriculares. Es decir, temas que engloban múltiples contenidos, que no pueden estar en una sola área del conocimiento y que es necesario que estén en todas las áreas, que permita el desarrollo personal integral de los alumnos, contribuyendo a que la sociedad sea mas respetuosa hacia la naturaleza. Las materias transversales confieren al currículo escolar una dimensión ética, vinculada a actitudes y valores de respeto, tolerancia, solidaridad, cooperación, propias de una sociedad democrática y no sólo de conocimientos.

La propuesta de estructuración de una sociedad moderna sustentable, se puede realizar sólo sobre la base de un amplio consenso social, dirigido al respeto a la naturaleza y sus ciclos regenerativos, a la voluntad de los pueblos, al desarrollo y protección de un medio ambiente más sostenible, a la solución de los problemas sociales básicos. La misión será estimular un proceso de unidad e integración de esfuerzos, voluntades y capacidades, donde el medio ambiente y la sociedad civil conforman una cadena necesaria.

La perspectiva ambiental como principio didáctico, debe estar siempre en el currículo, el diseño, su desarrollo, en su aprendizaje y evaluación. Esto permite al educando profundizar en la incorporación de la educación ambiental al sistema educativo y orientar las iniciativas concretas de enseñanza que faciliten la superación de las limitaciones que han caracterizado a muchas experiencias.

Sin embargo, han quedado pendiente una serie de aspectos importantes en lo educativo (curricular) como la falta de unas pautas metodológicas acordes con los planteamientos de educación ambiental, la ausencia de un marco teórico que integre los conocimientos escolares de carácter ambiental, casi nunca se han establecido mecanismos adecuados para valorar y trabajar contenidos, apropiados de educación ambiental, los aspectos afectivos, actitudinales, axiológicos y comportamentales.
Actualmente, encontramos en la práctica educativa varias modalidades de incorporación de la educación ambiental al currículo, que muestran diversas gradaciones, desde las que la consideran como un recurso didáctico más, hasta las que intentan definirla como un principio didáctico, articulador de diferentes elementos curriculares, ejemplo:

- $\quad$ Modelo de investigación del medio: en algunos casos, la educación ambiental no se integra realmente al currículo, se enfoca como una aproximación a la temática ambiental, que se realiza con los alumnos, sin modificar la dinámica del proceso de enseñanza-aprendizaje: los contenidos del saber educativos no están vinculados, sin criticidad, son sólo un recurso didáctico básico.

- Modelo de aproximación a partir de las disciplinas: la educación ambiental se asimila a un área del saber científico y los contenidos ambientales se incorporan al programa de una o varias disciplinas. Es un enfoque más académico, sin una perspectiva integrada del medio: predomina lo biológico o social, con sesgo cientificista o academicista.

Didácticamente, la educación ambiental reelabora un nuevo marco teórico y metodológico propio. Al trabajar un problema de contaminación ambiental, considera:

- contenido conceptual y componentes afectivos e ideológicos (forma de vida).

- $\quad$ adopta perspectiva global de conceptos generales (sistema, interdependencia).

- estudia el medio como un recurso didáctico.

- emplea una metodología de trabajo crítico y solidario.

detecta y resuelve problemas ambientales a nivel escolar. 
La educación debe incorporar el debate y discusión sobre una visión que supere las visiones parciales de técnicos especializados, carentes de conjunto. La finalidad de la educación ambiental en el currículo de la educación debe responder a la necesidad de que los individuos y grupos sociales adquieran con la ayuda de la actividad escolar, la capacidad de hacer frente, con actitud abierta, relativizadora, autónoma y negociadora, a los problemas que el medio socio-natural plantea.

La educación ambiental debe propiciar un cambio de actitudes y una participación responsable en la gestión del medio y crear actuaciones adecuadas con el entorno. La conceptualización del medio y las actitudes con respecto a la educación ambiental guardan estrechas relaciones. Hay que evitar una visión antropocéntrica de la intervención humana en el medio y la simple conservación, como único tipo de relación. Muchas iniciativas consideradas ambientales, se centran en un planteamiento de conservación del medio natural. $\mathrm{O}$ de mantener inalterable el medio, desde un ángulo de conservar es bueno, modificar es malo. La idea de conservación debería compatibilizar las ideas de permanencia y de cambio, en una visión más global del conjunto de elementos del medio, concebido como un sistema que puede adquirir diversos estados. La toma de decisión está impregnada de valores. Es importante conocer, valorar y disfrutar del medio en que se integra el colectivo humano.

Entre los objetivos para interrelacionar lo actitudinal con lo conceptual, en la educación ambiental están:

- desarrollar la sensibilización ante problemas socio-ambientales, mejorando la capacidad de plantearse problemas, de debatirlos, de construir opiniones propias, capaz de difundir esas problemáticas y sus propuestas de solución.

- adquirir conciencia de posibles incidencias de las propias actitudes $\mathrm{y}$ comportamientos, sobre el equilibrio del entorno, valorando las repercusiones de impactos importantes.

- avanzar en una comprensión más compleja, de conceptos básicos del funcionamiento del medio a ideas de interacción, cambio y dependencia del individuo con el medio.

- desarrollar actitudes de solidaridad entre los habitantes, a partir de la comprensión de las interrelaciones existentes entre los diversos medios y la interdependencia socio-económica, política y cultural del mundo, superando la separación rígida: medio natural y medio social.

- valorar el patrimonio cultural como expresión de la propia sociedad en que se vive, de respeto hacia las diversas manifestaciones culturales.

- a partir de un conocimiento y dinámica del medio, desarrollar la actitud y capacidad de disfrutar el medio, en equilibrio entre el uso individual y colectivo.

- desde la perspectiva global de una ética ambientalista, mejorar la capacidad y posibilidad de análisis, actitudes y comportamientos a la vida cotidiana, ambientalizando las acciones personales diarias y su integración en la sociedad.

El conocimiento escolar exige una convergencia de los campos del saber implicados, con la construcción de un marco de referencia didáctico que integre la reflexión psicológica, epistemológica y sociopolítica. Y no la mera conceptualización al aula.

\section{Importancia de la educación ambiental: componentes}

La educación ambiental promueve procesos orientados a la construcción de valores, conocimientos y actitudes que posibiliten formar capacidades que conduzcan hacia un desarrollo sustentable basado en la equidad y justicia social, el respeto por la diversidad biológica y cultural. 
La educación ambiental, es educación sobre cómo continuar el desarrollo al mismo tiempo que se protege, preserva y conserva los sistemas de soporte vital del planeta. Esta es la idea detrás del concepto de desarrollo sustentable.

Para eso, la educación ambiental en el aula es transdisciplinaria, desde la perspectiva científica (ecología, biología), ideologista (valores, comportamientos, actitudes) e integradoras (aprendizaje, educación). El cambio exige la transición de una concepción simple a una más compleja del medio, supone reestructurar las propias creencias del pensamiento cotidiano. Con propuestas de carácter integrador y globalizador, donde la educación ambiental desarrolla una visión del mundo más ambiental.

Entre los componentes de la educación ambiental, se puede pensar que la educación ambiental consiste de cuatro niveles diferentes:

\section{a. fundamentos ecológicos}

Este nivel incluye la instrucción sobre ecología básica, ciencia de los sistemas de la Tierra, geología, meteorología, geografía física, botánica, biología, química, física, etc. El propósito de este nivel de instrucción es dar al alumno informaciones sobre los sistemas terrestres de soporte vital. Los científicos han descubierto muchas reglas ecológicas. Pero, muchas conductas humanas y decisiones de desarrollo se violan.

\section{b. concienciación conceptual}

De cómo las acciones individuales y de grupo pueden influenciar la relación entre calidad de vida humana y la condición del ambiente. Es decir, no es suficiente que uno comprenda los sistemas de soporte vital (reglas) del planeta; también debe comprender cómo las acciones humanas afectan las reglas y cómo el conocimiento de estas reglas pueden ayudar a guiar las conductas humanas.

\section{c. investigación y evaluación de problemas}

Esto implica aprender a investigar y evaluar problemas ambientales. Debido a que hay demasiados casos de personas que han interpretado de forma incorrecta o sin exactitud asuntos ambientales, muchas personas se encuentran confundidas acerca de cual es el comportamiento más responsable ambientalmente. Por ejemplo, ¿es mejor para el ambiente usar pañales de tela que pañales desechables? ¿Es mejor hacer que sus compras la pongan en una bolsa de papel o en una plástica? Muy pocas veces las respuestas a tales preguntas son sencillas.

\section{d. capacidad de acción}

Este componente enfatiza el dotar al alumno con las habilidades necesarias para participar productivamente en la solución de problemas ambientales presentes y la prevención de problemas ambientales futuros. También se encarga de ayudar a los alumnos a que comprendan que, frecuentemente, no existe una persona, agencia $u$ organización responsable de los problemas ambientales.

Los problemas ambientales son frecuentemente causados por las sociedades humanas, las cuales son colectividades de individuos. Por lo tanto, los individuos resultan ser las causas primarias de muchos problemas, y la solución a los problemas probablemente será el individuo (actuando colectivamente).

El propósito de la educación ambiental es dotar a los individuos con:

conocimiento necesario para comprender los problemas ambientales;

- desarrollo de habilidades necesarias para investigar y evaluar la información disponible sobre los problemas;

- desarrollo de capacidades necesarias para ser activo e involucrarse en la 
resolución de problemas presentes y la prevención de problemas futuros; $y$,

- desarrollo de habilidades para enseñar a otros a que hagan lo mismo.

\section{Educación ambiental y conocimiento}

El conocimiento cotidiano va unido a la solución de problemas prácticos del sujeto. Su enriquecimiento, en la construcción de una nueva visión, permite enfrentar la realidad:

- Considera todas aquellas formas de conocimiento educativo: el científicotecnológico, las ideas que empleadas y la práctica de grupos ecologistas a la propia problemática socio-ambiental.

- Los fines que persiguen estos conocimientos, seleccionar y delimitar los contenidos más relevantes: que lo aprendido sea útil para las relaciones humano-ambiente; coherente de ideas y significativo para el alumno (hacerlo suyo).

- Luego de seleccionar y organizar los contenidos, convertir ese conjunto de conocimientos en una propuesta general de conocimiento escolar integradora de lo científico, ideológico, cotidiano y crear una nueva visión del mundo. Aquí adquiere importancia la aplicación constructivista, holística y sistémica.

Los conocimientos deben enlazarse en tres formas con la problemática ambiental: ideológico, científico y cotidiano. Luego, concretar la propuesta general en tópicos y objetivos de estudio a trabajar.

Los contenidos de la educación ambiental tienen las siguientes características:

- El conocimiento del medio se identifica con las disciplinas concretas (biología, geografía), donde la temática ambiental es una reflexión sobre como entender y actuar en el mundo.

- No se debe identificar con un conocimiento cerrado, estático, único de la realidad. La educación ambiental se basa en un sistema general de ideas, que va de lo concreto a lo general, de lo simple a lo complejo, en los diferentes niveles educativos. Que desarrolle una manera de interpretar el mundo global, abierto y flexible, diverso.

- La construcción del sistema educativo supone la transición de lo inmediato a lo lejano, la comprensión de la causalidad compleja, la superación de la visión antropocéntrica del mundo. Estos conocimientos deben estar siempre en los contenidos de educación ambiental.

- De la complejidad y abstracción a una visión sincrética (realidad como un todo homogéneo), a una visión analítica (realidad como suma de partes y relaciones sencillas), a una visión sistémica (realidad como una red de interacciones, con jerarquía de sistemas unos con otros).

- Pasa de un enfoque descriptivo de la realidad, a una explicación de causalidad lineal de esta a la interacción y explicación multicausal.

- La comprensión de interacción: un determinado sistema, con diversidad y noción del equilibrio dinámico, de lo simple a lo complejo.

Una comunidad de aprendizaje es un proyecto de transformación social y cultural de un centro educativo y de su entorno, para conseguir una sociedad de la información para todas las personas. Está basada en el aprendizaje dialógico y en una educación participativa de la comunidad en todos los espacios, incluida el aula. La participación es un elemento base, dentro del proyecto educativo y es donde el papel de los educadores y educadoras sociales toma un nuevo sentido. Estos trabajan de igual a igual con familiares, voluntariado, profesorado y con todas las personas implicadas en el proyec- 
to, con un objetivo común: que todos tengan igualdad de oportunidades y resultados en la sociedad de la información.

La educación ambiental como un conocimiento integrado del medio socionatural, donde la mente es un sistema activo-participativo, donde todo está en relación con todo, como un proceso abierto, flexible y creativo, para la solución de problemas socio-ambientales. Es necesario un enfoque sistémico, donde los objetos de la realidad son sistemas organizados, integrados $\mathrm{y}$ jerarquizados unos con otros. Trascender la lógica, mediante el vínculo naturalezasociedad, con un enfoque integrado de ambas, entre ciencias naturales y sociales, donde se concreta el conocimiento:

- Valoración ambiental (no económica) del medio natural.

- Relaciones ecológicas y la participación del ser humano.

- Paisaje y sensibilización estética y conservación del medio.

- Impacto producido por el ser humano sobre el medio y sus formas de producción.

- Concienzación ante la problemática ambiental.

- Negociación en el análisis y resolución de conflictos entre humanos.

- Fomento de hábitos por una mejor calidad de vida.

- Consumidores y usuarios activos y críticos.

El conocimiento sobre el medio natural debe organizarse como una visión de mundo, donde los conceptos, actitudes y procedimientos forman tramas de vida, donde todo interactúa con todo. El proceso de construcción del conocimiento supone la transición desde una visión simple a una compleja del medio, mejor organizado y jerárquico en tramas e hipótesis de progresión, dentro del proceso de enseñanzaaprendizaje, que refleja el contenido:

Naturaleza causal de las relaciones ecológicas: no se alude a relaciones causales, solo se describen los elementos presentes, con relaciones de dependencia.

- Relaciones ecológicas se interpretan como relaciones en un mismo y único sentido (causalidad lineal) y de forma simple.

- Reconoce las interacciones e interdependencia entre elementos que se relacionan.

- Reconoce que las interdependencias presentes en los ecosistemas generan procesos de retroalimentación (causalidad circular).

- Este enfoque ve más lo dinámico que los estáticos.

En la construcción del sujeto, se pasa desde una concepción analítica del medio (realidad como suma de partes) a una visión sistémica (realidad como una jerarquía de sistemas unos con otros). Se sustituye el enfoque descriptivo de la realidad (que desconoce las causas de las cosas) por la búsqueda de explicaciones causales, lineales (un factor del medio determina a otro) y luego interactivas (los factores se determinan mutuamente). Esta progresión va de lo simple (un animal come a otro) a lo complejo (redes tróficas, ciclos), con la superación del mundo egocéntrico (todo esta en función de uno) y el antropocéntrico (la vida desde la óptica humana) y sociocéntrico (la realidad se analiza desde la óptica socio-natural del sujeto, sin considerar la diversidad natural).

El conocimiento cotidiano esta ligado a la acción, ve lo evidente e inmediato, no lo oculto, centrado en lo episódico y particular, con una visión de antropocéntrica, con causalidad simple, la idea de un mundo aditivo (suma de elementos), un orden de las cosas, estático, rígido, dogmática, sin ver diversidad, con una ideología basada en la explotación, dominio, competencia e individualismo.

La educación ambiental supone una metodología, que permita abordar el estudio de problemas ambientales de manera 
integral, para trabajar contenidos científicos y cotidianos. Esta metodología se basa en el planteamiento de problemas y en su trabajo a lo largo de la secuencia de aprendizaje. La metodología cuenta con una importante experiencia de aplicación de innovaciones didácticas, ha resultado una alternativa al activismo (desconexión con procesos de aprendizaje). Esta metodología es adecuada para la educación ambiental, ya que se basa en la idea de investigación del alumno e integra los contenidos comportamentales y actitudinales con los conceptos, aspecto que favorece el trabajo con propuestas ambientales. Destaca la coincidencia entre el planteamiento y tratamiento de problemáticas ambientales $\mathrm{y}$ el carácter de investigación de situaciones problemáticas, propia de esta metodología. El abordar a diario situaciones problemáticas contribuye a nuevos conocimientos, de forma que puede aprender en la medida que trabaja con esas problemáticas y elabora respuestas (cognitivas, afectivas, conductuales). La investigación es considerada como una estrategia básica de conocimiento y actuación en la realidad, propia del comportamiento del ser humano.

Con esta metodología, el alumno se plantearía qué entiende por problema (ambiental), ya que será su eje de organización y tratamiento de los contenidos escolares, ha de tener una clara dimensión ambiental y social, como relevancia educativa de esos problemas y presentar una formulación cercana al mundo en el que se mueven los alumnos.

\section{Conclusión}

Ante los nuevos paradigmas educativos y la crisis socio-ambiental, la educación ambiental constituye una alternativa o solución dirigida a lograr una acción educativa, que convierta al individuo en sujeto de su propio destino, comprometido con su sociedad y conciencia de los problemas ambientales, buscando soluciones necesarias para un desarrollo ambiental sustentable. Es importante destacar el papel fundamental que desempeña la sociedad en el logro de estos propósitos, promoviendo la capacidad de decisión, autonomía y gestión propia, dirigida a las asociaciones comunales, cooperativas, grupos culturales, movimientos ecologistas y todas aquellas fuerzas sociales que trabajan por un desarrollo alternativo o sustentable.

La educación ambiental representa una solución o alternativa básica a la problemática ambiental. La dominación interna/ externa a que está sometida nuestra realidad social, impide el surgimiento de toda conciencia crítica en el individuo. Por eso, se requiere de alternativas educacionales que permitan romper con estos lazos de dependencia y gestar una de intereses nacionales. Se deben promover nuevos estilos pedagógicos que conduzcan a la revalorización del individuo como ser social, cultural, independiente y capaz de potenciar cambios en la sociedad postmodernista.

La educación ambiental no es neutra, es una alternativa a la problemática ambiental. La dominación interna/externa a que está sometida nuestra realidad, impide el surgimiento de toda conciencia crítica. Por eso, requiere de alternativas educativas comprometidas, que rompan con los lazos de dependencia y gesta una de intereses nacionales.

La educación ambiental ante la crisis económica y social que vive nuestra realidad, representa una opción viable para promover cambios sociales, nuevos conocimientos, crear conciencia de participación, de equilibrio social y ecológico, de desarrollo sostenible y de comportamientos culturales, que hagan real una sociedad más igualitaria y solidaria, con objetivos interrelacionados para moldear una nueva dimensión del ser humano. Por ello, la educación ambiental, concebida bajo una visión integral, basada en una pedagogía liberadora será capaz de generar un pensamiento crítico, consciente y reflexivo en el individuo, que permita abrir caminos hacia 
alternativas que se deriven en procesos de transformación social y más justa distribución de la riqueza social, para erradicar el hambre, analfabetismo, miseria. Se debe eliminar el énfasis mercadocéntrico y aplicar el concepto: la coexistencia humana con y dentro de la naturaleza.

La educación ambiental todavía no esta dotada pedagógicamente, del instrumental necesario para plantear cognoscitiva, epistemológica ni transformativa la interrelación histórica de sociedad y naturaleza. No se instruye debidamente sobre la naturaleza, ni aquellas estructuras y prácticas sociales que articulan a la sociedad con el ambiente natural. Aunque, en algunos centros superiores se hacen los primeros intentos en los planes y programas de estudios. Estamos en un largo periodo de transición, donde la naturaleza no espera y la sociedad no se percata. Todavía, el educador no esta preparado política, educativa y ambientalmente para llevar una relación mas objetiva y consciente con el ambiente natural. Debido a que las relaciones de dominación e intereses particulares atentan contra la naturaleza, la sustentabilidad y el bienestar humano colectivo. Desde este ángulo, las perspectivas del estudio ambiental se caracterizan, por no encajar en un currículo académico convencional.

Sin embargo, la educación ambiental no se ha incluido ni aplicado como materia transversal, ni transdisciplinaria en los actuales diseños curriculares.

La educación dentro de un proceso de globalización está fuera de un contexto ambiental. Las políticas educativas sobre medio ambiente son casi retóricas, visto desde un análisis y comparación con la realidad socio-natural. Los obstáculos que enfrenta la educación ambiental son básicamente teórico-metodológico: deficiencias de las ciencias sociales frente a lo ambiental (clima, vegetación, producción, reforestación); deficiencia de la ciencia social frente a lo social: aspectos de la economía, riqueza, pobreza; políticas: planes y modelos de desarrollo, estructuras administrativas.
Hay un divorcio de las ciencias sociales y naturales respecto a la realidad (social y ambiental) que se ha expandido y ha cambiado. Además, trae vicios intrínsecos de las mismas ciencias; ejemplo, se da la separación del sujeto del objeto y una concepción evolucionista, como explicación de desarrollo social y natural; obstruyendo así, en la educación, los estudios socioambientales.

La realidad se fragmenta en diversas ciencias, generando híbridos, por lo que no hay una sola teoría ambiental socialmente, sólo conceptos no aplicados realmente, como desarrollo sostenible, donde el discurso no va con la realidad, al igual que los conceptos de democracia, libertad e igualdad.

La (de)formación académica de profesionales ambientales, limitan la aplicación de metodologías participativas activas; lo que sobresale la falta de profundidad, precisión y sistematización conceptual y teórica. Con facilidad se pierde, la concepción del carácter histórico en la relación sociedadnaturaleza, lo que dificulta la comprensión de las tendencias y cambios de épocas y surgimiento de nuevos paradigmas. El educador ambiental tiene una incapacidad epistemológica y teórico-metodológica para comprender y tratar debidamente la relación sociedad-naturaleza.

La educación ambiental es una educación crítica, implica que el ser humano y la naturaleza están en constante interacción mutua. La clave del discurso ambiental es comprender el quehacer del individuo y sociedad ubicado en el tiempo y espacio, como un sujeto que transforma al mundo, para su propio beneficio, sin alterar el equilibrio con la naturaleza; sino, se autodestruye.

La educación ambiental, esta en proceso de formación, avanza a través de la solución a los nuevos paradigmas humanísticos que buscan evolucionar y adaptarse a las nuevas circunstancias y problemas actuales, como el deterioro ambiental y social.

La educación ambiental no es un simple conjunto de conocimientos, sino una 
actitud creativa y práctica diaria hacia la comprensión y transformación del proceso histórico, su ubicación en el cosmos, el pensamiento humano y su nivel socio-económico y relación ambiental. Los estudios ambientales buscan replantear dentro del marco histórico y su relación de dominación tradicional, una visión filosófica hacia el ambiente y sociedad. A través de los diversos enfoques pedagógicos (didácticos) plantea nuevas estrategias metodológicas interdisciplinarias en los procesos de enseñanza-aprendizaje, sobre aspectos sociales y ambientales dialécticamente: valores ambientales, nuevos estilos de vida, más justos, equitativos y sustentables.

En lo ambiental se debe evitar la deforestación, la contaminación y el deterioro ambiental general; estimular una producción con respeto al ambiente y la sociedad, aplicando tecnología limpia y una revalorización económica de los recursos naturales (eliminar las externalidades de la economía globalizada), con desarrollo e integración al medio ambiente (flora y fauna, ecosistemas).

El desarrollo insustentable no es resultado de una mala planificación o ejecución de un proyecto, ni falta de visión o mala estructuración institucional. La crisis socio-ambiental NO es un problema técnico, ni tecnológico, ni económico, ni social; es ético, moral, es político, con sus estructuras de relaciones deshumanizadas (socio-político), que enfatizan en mercados desregularizados.

El desarrollo dominante no comparte de modo equitativo, ni toma en cuenta las necesidades locales, para eliminar la pobreza y el hambre debidamente.

La educación ambiental plantea un nuevo desarrollo socio-económico alternativo, un cambio estructural radical, toda una revolución, donde lo ambiental sea eje, a través de la autogestión de las comunidades, desconcentración, participación activa, descentralización, democratización y transformación de las relaciones de poder, donde el entorno predominante nacional (institucional) e internacional (financiero) y la economía de mercado regularizada tome en cuenta lo natural y social.

La sociedad debe ser más justa, equitativa y asumir un desarrollo sustentable, basado en los principios de equidad social, respeto a la integridad ecológica de los ecosistemas, modelo económico alternativo y democracia participativa.

\section{Referencias bibliográficas}

Ander-Egg, E. (1994). El desafío ecológico. San José: EUNED.

Beck, U. (1998). La sociedad de riesgos: hacia una nueva modernidad. Barcelona: Paidós.

Boff, L. (1997). Ecología: grito de la Tierra. Grito de los pobres. Barcelona: Ed. Trotta.

Boyden, S. (1987). Western civilization in biological perspective. Oxford: Clarendon Press.

Capra, F. (1997). El nuevo paradigma ecológico. En V. Amador Zúñiga y S. Ramírez Gatgens (Comp.), Antología Humanismo y Medio Ambiente (pp.: 9-13). San José: CEG-UNA.

Castro, G. (1994). Los Trabajos de Ajuste y Combate: naturaleza y sociedad en la historia de América Latina. Cuba: Casa de las Américas.

Charpantier, C., Zúñiga, M., Hernández, L., Vargas, G. y Zúñiga, C. (1999). La dimensión ambiental como eje transversal, un reto para las universidades estatales costarricenses: el caso de la formación de docentes de primaria. Innovaciones Educativas, 11, Año VI, 61. 
Freire, P. (1975). Pedagogía del oprimido. Río de Janeiro: Editorial Paz y Tierra.

Gudynas, E. (1998). Globalización, políticas sociales y medio ambiente. Tareas, Enero-Abril, 98, 23-44.

Guier Serrano, E. (2002). Educación ambiental en Costa Rica: tendencias evolutivas, perspectivas y desafíos. San José, Costa Rica: EUNED.

Kuri, G. A. (1997). La globalización: hacia un nuevo tipo de hegemonía. En V. Amador Zúñiga y S. Ramírez Gatgens (Comp.), Antología Humanismo y Medio Ambiente (pp. 80-85). San José: CEG-UNA.

García, J., Guier, E. y Chacón, I. (Eds.) (2000). Antología ambiente: problemática y opciones de solución. San José: EUNED.

Leff, E. (1986). Los problemas del conocimiento y la perspectiva ambiental del desarrollo. México: Siglo XXI.

Leff, E. (1994). Ecología y Capitalismo. México: Siglo XXI.

Martínez, R. (1998). Reflexión sobre educación, sociedad y ambiente. Revista Tópicos del Humanismo, 41, s. p.
Martínez, R. (2001). El paradigma socioambiental. Revista Espiga, 1, Año 1, 129, 138.

Martínez, R. (2003). Alternativa para un desarrollo sustentable. Revista InterSedes, 7(IV), 11-25.

Mires, F. (1990). El discurso de la naturaleza. Ecología y política en América Latina. Costa Rica: DEI.

Montoya, F. (1993, Diciembre). La antropología ambiental: una rama verde. Revista de Ciencias Sociales, 62, 103-117.

Mora, E. (1994). Claves del discurso ambientalista. Heredia: Editorial Fundación UNA.

Stokke, O. (1994). Los dilemas del desarrollo sostenible. Revista FLACSO, 63, 14 .

Toledo, V. (1990). Modernidad y ecología: la nueva crisis planetaria. Ecología Política, 3, 9-22.

Vargas, G. (1993). La relación hombremedio ambiente. Revista Umbral 3(9), septiembre, 3-9. 